http://jmscr.igmpublication.org/home/ ISSN (e)-2347-176x ISSN (p) 2455-0450

crossref DOI: https://dx.doi.org/10.18535/jmscr/v8i10.42

\title{
Management of Recurrent Shoulder Dislocation by Arthroscopic Repair
}

\author{
Authors \\ Mudidana Prudhvi ${ }^{1}$, Moka Siddartha ${ }^{2}$ \\ ${ }^{1}$ Junior Resident, Department of Orthopaedics, Andhra Medical College \\ ${ }^{2}$ Senior Resident, Andhra Medical College
}

\begin{abstract}
Background: The shoulder joint, by virtue of its anatomy and biomechanics, is one of the most unstable and frequently dislocated joints in the body, accounting for nearly 50\% of all dislocations, with a $2 \%$ incidence in the general population.

The cases are treated usually either open bankart's repair and arthroscopic repair since early 19th century, but there are reports of the more incidence of failures after arthroscopic repair than open repair. But in recent period with understanding of the anatomy and pathophysiology behind dislocation and advent of newer technique and implants, favourable reports are seen in recent period.

Patients and Methods: This study is conducted in the department of orthopaedics, Andhra medical college, Visakhapatnam, Andhra Pradesh from July 2017 to December 2017.

The recurrent anterior shoulder dislocations patients presented to outpatient department and emergency department during the period of study fulfilling the inclusion criteria are included in the study.

All the patients are treated with arthroscopic bankart repair using suture anchors and followed up for a period of 1 year to assess the functional outcome.

Results: Excellent results are achieved in 16 patients and good results seen in 3 patients. No complications are reported during the study period.

Conclusion: Arthroscopic bankart repair with suture anchors are a good option for treating the recurrent anterior shoulder dislocation with good functional outcome.
\end{abstract}

\section{Introduction}

The shoulder joint, by virtue of its anatomy and biomechanics, is one of the most unstable and frequently dislocated joints in the body, accounting for nearly $50 \%$ of all dislocations, with a $2 \%$ incidence in the general population.

The incidence of glenohumeral instability has been estimated at 8.2 to 23.9 per100,000 personyears. The incidence in at-risk populations is significantly higher (military population, 1.69 per 1000 person-years; athletes, 0.12 injuries/1000 athletic exposures)
The glenohumeral joint is the most commonly dislocated joint in the body representing $45 \%$ of all dislocations. Reported instability rates range from 11.2 to $23.9 / 100,000$ person- years and likely underestimate the true incidence as the data is defined by patients seeking medical attention This topic is chosen as there were different results in various studies reported throughout the world. Though frequently treated surgically in our geographical area, there are very few studies reported through standard journals and less number of studies as thesis. In this study we have 
made an attempt to study the incidence, age group, post operative stability, range of movements and post operative subluxation and dislocation rate.

\section{Aims and Objectives}

1. To study the post operative stability and range of movements after arthroscopic bankart's repair

2. To study the post operative complications, failures of surgical procedure manifested as dislocation during the study period

\section{Patients and Methods}

The present study on management of recurrent anterior shoulder dislocation by arthroscopic bankart repair, prospective study is carried out in the department of orthopaedics , Andhra medical college, king George hospital from July 2017 to December 2017.

All Patients are those presented to our outpatient department during the study period with recurrent episodes of anterior shoulder dislocation who gave consent willingly for thorough clinical evaluation, laboratory evaluation and surgical management are involved in the study.

All the patients are initially stabilized with closed manipulation and reduction. Total Sample number -were19 pateints, out of which 18 male patients, 1 female patient

The cases treated are followed prospectively after surgery for a period of 12 months and range of movements are assessed. All the cases are recurrent anterior dislocation of shoulder with initial episode occurring following trauma in road traffic accidents, sports activity, other miscellaneous injuries.

\section{Inclusion Criteria}

1. Age between $15-40$ years

2. 2-5 episodes of dislocation

3. Anterior shoulder dislocation

4. Actively mobile patients

5. Documented bankart lesion in MRI

\section{Exclusion Criteria}

1. AGE less than 15 years and above 40years

2. First time dislocations
3. Chronic dislocations

4. Generalized ligamentous laxity, multidirectional instability

5. Epilepsy

6. Other types of dislocations

7. Associated fractures of shoulder girdle

8. Peripheral nerve injuries: Brachial plexus or axillary nerve

9. Significant $(>25 \%)$ Humeral head deficiency or $(>20 \%)$ erosional glenoid deficiency

10. Associated rotator cuff, biceps pathology and labral pathologies

11. Smokers and alcoholics

\section{Pre Operative Assessment}

All the patients are initially stabilized with analgesic and conforming anterior dislocation clinically with different signs of dislocation and confirmed with anteroposterior radiograph. closed manipulation and reduction of the anteriorly dislocated shoulder by kocher's maneouver without aneasthesia following which patients were immobilized in arm sling in internal rotation

Stability of the shoulder is assessed by the apprehension test, Jobe's relocation test, sulcus test, load and shift test, drawers test, and Gagey hyperabduction test to assess for anterior deficiency of glenoid, ligamentous laxity and inferior capsular laxity respectively.

Plain radiograph anteroposterior with 15degree internal rotation are taken to assess the Hill Sach's lesion on the humerus and MRI shoulder to document evidence of bankart lesion in all the cases were taken.

\section{Arthroscopic Bankart Repair Technique}

Glenoid bone loss greater than $6 \mathrm{~mm}$ should be restored with an open laterjet procedure.

While visualizing from the anterosuperior portal, use an elevator to free up the capsule down to the subscapularis muscle, which should be visible. Abrade the glenoid neck to stimulate healing and from same portal if necessary, perform a capsular plication procedure posteriorly, extending along to 
the attachment of the posterior band of the inferior glenohumeral ligament.

Using a rasp, freshen the soft tissue and the intended area of plication to incite some inflammation without damaging the tissue. Use a suture shuttle to pass PDS sutures, starting at about the 6-o'clock position and taking a bite of approximately $1 \mathrm{~cm}$ of capsule in a pinch-tuck
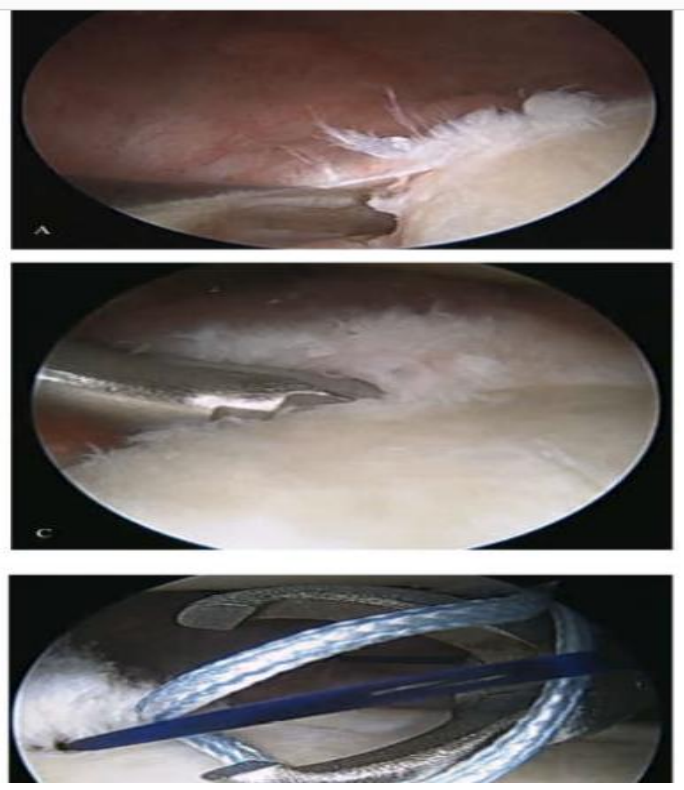

During the follow up patient is assessed outcome was measured using the ROWE SCORE for shoulder instability, it includes measurement of three parameters

1) Stability

2) Range of Motion

3) Function

ROWE scoring system score

Stability

No recurrence, subluxation or apprehension 50

Apprehension when placing arm in certain positions 30

Subluxation 10

Recurrent dislocation 0

\section{Range of motion}

$100 \%$ normal ER, IR and elevation 20

$75 \%$ normal ER, IR and elevation 15

$50 \%$ normal ER, IR and elevation 5

$50 \%$ normal elevation and IR, No ER 0 technique, making sure that the needle comes out through the capsule and passes up under the labrum in its appropriate position. It is easier to pass multiple sutures first, store them outside the cannula, and tie them later. Generally, three sutures are passed, with the upper extent being at the attachment of the posterior band of the inferior glenohumeral ligament
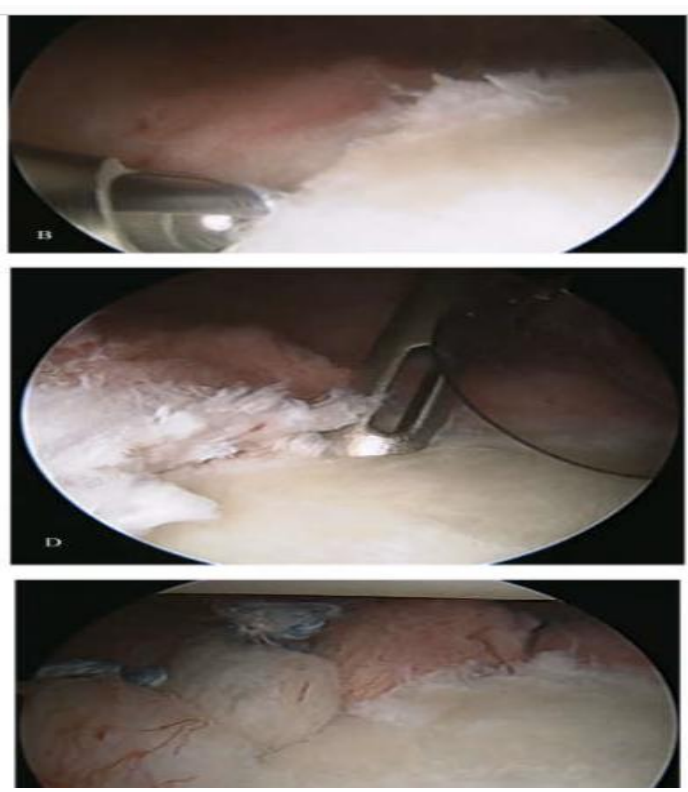

\section{Function}

No limitation of work /sports, little/no discomfort 30

Eg. shoulder strong overhead, lifting, throwing

Mild limitation and minimal discomfort 25

Moderate limitation and discomfort 10

Marked limitation and pain 0

Final scores were based on the combined point scores from the three categories of the evaluation. Results are designated as excellent, good and fair poor.

Excellent 90- 100 points

Good 75 - 89 points

Fair 51 - 74 points

Poor $<50$ points

\section{Results}

All the cases were followed until a period of 1 year and the range of movements assessed at each follow up visit and the functional status assessed by ROWE score and Results were analysed. 


\section{Post operative ROWE SCORE}

At 6 months

13 patients had score of 80

5 patients had score 75

1 patient had score 65 .
At 1 year:

16 patients excellent results ( $84.21 \%$ cases) with post operative score of

[95 in 11 patients, 90 in 5 patients]

3 patients had good outcome with score 80 $(15.79 \%)$

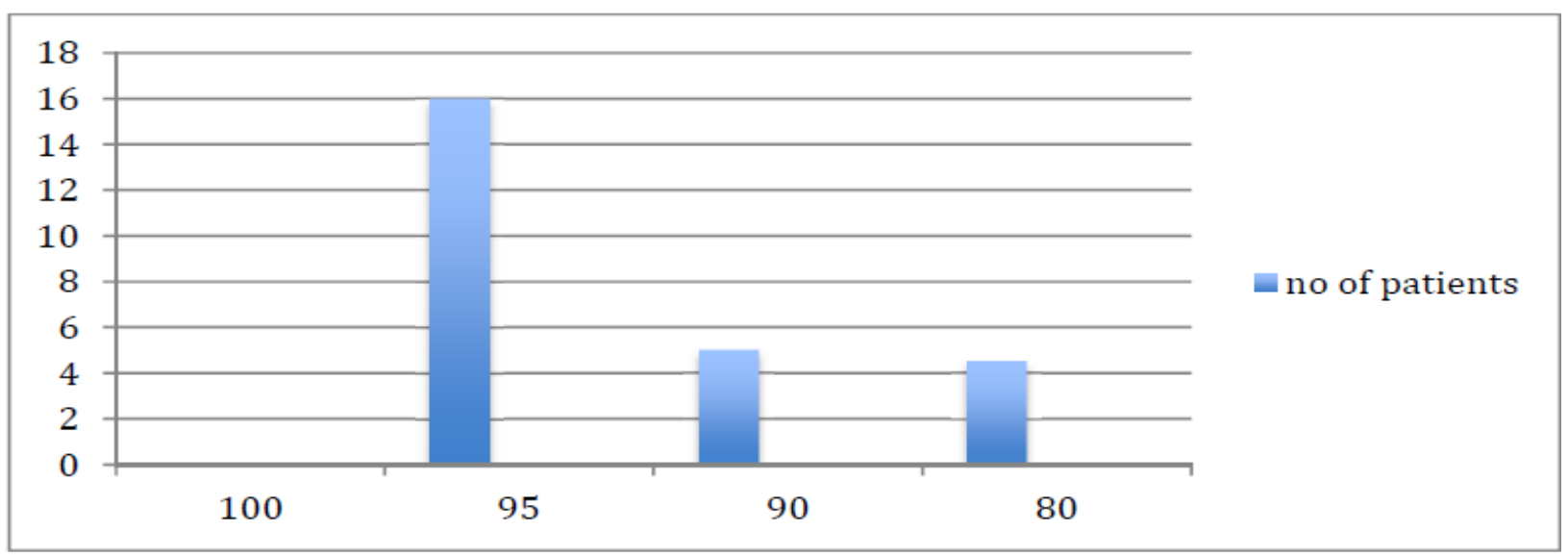

\section{Discussion}

Arthroscopic shoulder stabilization for recurrent glenohumeral instability has evolved considerably over the past two decades. Arthroscopic stabilization can potentially provide more favorable range of motion and early return to fullfunctional activity in high-demand patients ${ }^{(1)}$

However, not always was the arthroscopic treatment considered an ideal solution in the approach of shoulder instability, mainly because of the high failure rate that was reported in early studies $^{(2,3)}$. However, in a meta analysis published in 2009, Brophy and Marx found that arthroscopic surgery using suture anchor fixation has redislocation rates similar to those of open surgery (6.4\% and $8.2 \%$, respectively).

\section{Statements from Various Studies Show Following Inferences}

Patients with recurrent shoulder dislocation had a higher arthroscopic degree of Injury. These patients presented more posterior labral lesions, SLAP tears, and rotator cuff pathology than patients with a first episode of shoulder dislocation $^{(4)}$

Delayed time to recurrence after open Bankart repair suggests that the open technique may be more suited to withstand the high stress and demands of a heavy duty profession (contact athletes and heavy manual labor) ${ }^{(5)}$.

Surgical treatment of anterior shoulder instability using arthroscopic suture anchor and open Bankart techniques yields similar long-term clinical outcomes, with no significant difference in the rate of recurrent instability, clinical outcome scores, or rate of return to sport ${ }^{(6)}$

Arthroscopic stabilization for anterior instability of the shoulder is a reliable procedure with respect to shoulder function, range of motion, and postoperative return to sports in contact and collision athletes. Compared with the contact group $(0 \%)$, the collision group yielded a higher failure rate $(14.7 \%)$.

In this retrospective study of a consecutive cohort of athletes, arthroscopic anterior shoulder stabilization significantly improved functional scores. However, only $60 \%$ of the athletes achieved the same level of competition, and there was a $20 \%$ recurrence rate.

Arthroscopic Bankart repair is an effective surgical option for traumatic shoulder instability in adolescents participating in collision and contact sports. At a minimum 4-year follow-up, arthroscopic Bankart repair effectively restored 
stability in $90 \%$ of cases; $80 \%$ returned to their preinjury level of sport. ${ }^{(7)}$

Clinical outcome at a mean follow-up of 13 years after arthroscopic repair of anterior-inferior shoulder instability is comparable with the reported results of open Bankart repair in the literature and allows management of concomitant lesions arthroscopically. Stabilization after the first-time dislocation achieves better clinical and radiological outcomes than after multiple dislocations.

\section{Conclusion}

It is concluded that Arthroscopic Bankart's repair in recurrent anterior shoulder dislocation with suture anchors is a reliable procedure with respect to satisfactory shoulder function, recurrence of dislocation and range of movements. Recurrent dislocation cases due to failure of the procedure are treated with either open or arthroscopic latarjet procedure.

\section{References}

1. Tjoumakaris F.P., Bradley J.P. The rationale for an arthroscopic approach to shoulder stabilization. Arthroscopy. 2011;27(October (10)):1422-1433.

2. Hobby J., Griffin D., Dunbar M., Boileau P. Is arthroscopic surgery for stabilisation of chronic shoulder instability as effective as open surgery? A systematic review and meta-analysis of 62 studies including 3044 arthroscopic operations. J Bone JtSurg Br. 2007;89:1188-1196

3. Sisto D.J., Cook D.L. Intraoperative decision making in the treatment of shoulder instability. Arthroscopy. 1998;14:389-394.

4. Brophy R.H., Marx R.G. The treatment of traumatic anterior instability of the shoulder: nonoperative and surgical treatment. Arthroscopy. 2009;25(3):298304
5. Clin Orthop Relat Res.2012 Apr;470( 4):961-4. doi: 10.1007/s11999-011-2229

6. Gutierrez V, Monckeberg JE, Pinedo M, Radice F. Arthroscopically determined degree of injury after shoulder dislocation relates to recurrence rate Orthop $\mathrm{J}$ Sports Med.2016 Jun 23;4 (6):2325967116654114. doi:10.1177/2325967116654114.2016 Jun.

7. Comparison of Time to Recurrence of Instability after Open and Arthroscopic Bankart Repair Techniques. Virk MS, Manzo RL, Cote M Arthroscopy.2013 May;29(5):920-33. doi: 10.1016/j.arthro.2012.11.010. Epub2013 Feb 5. 